\title{
Résidus de pesticides organochlorés chez les bivalves et les poissons de la lagune de Moulay Bousselham (Maroc)
}

\section{Benbakhta Bouchaib ${ }^{1 *}$, Fekhaoui Mohamed ${ }^{2}$, El Abidi Abdellah ${ }^{1}$, Idrissi Larbi ${ }^{1}$, et Lecorre Pierre ${ }^{3}$}

${ }^{1}$ Institut national d'hygiène, Département de toxicologie, Av. Ibn Batouta, Agdal, BP 769, Rabat, Maroc

${ }^{2}$ Laboratoire d'hydrobiologie et écotoxicologie, Institut scientifique, Rabat, Maroc

${ }^{3}$ Laboratoire de chimie marine, Institut universitaire européen de la mer (IUEM), 29280 Plouzané, Brest, France.

(Reçu le 28 Juin 2006, accepté le 02 Décembre 2006)

*Correspondance, courriel : benbakhta@yahoo.fr

\section{Résumé}

Le présent travail consiste à évaluer le degré de contamination par les pesticides Organochlorés (POC) des palourdes (Venerupis deccusatua), des moules (Mytilus edulis) et des mulets (Mugil cephalus) collectés au niveau de la lagune de Moulay Bousselham. 12 pesticides organochlorés sont considérés dans cette étude: DDT et ses isomères (DDD et DDE), $\mathrm{yHCH}$ et $\mathrm{aHCH}$, aldrine, endrine, diéldrine, heptachlor, heptachlor epoxide, hexachlorobenzene (HCB) et $\alpha$ endosulfan.

L'étude a montré une contamination non négligeable par les pesticides organochlorés. Le DDT et ses métabolites présentent les concentrations les plus importantes chez toutes les espèces. Le lindane et ses isomères sont également présents mais à des faibles doses. Les cyclopentadiènes se trouvent sous forme de traces.

Chez les palourdes, les concentrations des pesticides organochlorés varient de 9,2 à $51.72 \mathrm{ng} / \mathrm{g}$ (poids sec) avec un maximum détecté au 
niveau de l'embouchure de l'oued Drader. Les moules étaient moins contaminés que les palourdes, les concentrations varient de 4.53 à 16.81 $\mathrm{ng} / \mathrm{g}$. La contamination du mulet par les POC est plus marquée avec des concentrations qui varient de $12.52 \mathrm{ng} / \mathrm{g}$ enregistrées au moi de novembre et $82.39 \mathrm{ng} / \mathrm{g}$ p.s détectées au mois de mai. La forte proportion du DDE par rapport au DDT montre qu'une bonne partie de la contamination est très ancienne.

Les quantités de pesticides organochlorés relevés chez les palourdes, moules et le mulet de la lagune de Moulay Bousselham ne dépassent pas les limites maximales admissibles dans les produits de pêche. La consommation de ces espèces ne posent pas actuellement de problème de santé publique.

Mots-clés : Organochlorés, pesticides, bivalves, poissons, lagune, Maroc.

\section{Abstract}

Oragnochlorine pesticide residues in bivalves and fishes from the Moulay Bousselham lagoon (Morocco)

This work involves an assessment of the degree of contamination by Organochlorine pesticides (POC) of clams (Venerupis deccusatua), mussels (Mytilus edulis) and fish mullet (Mugil cephalus) from the Moulay Bousselham lagoon. 12 organochlorine pesticides are considered in this study: DDT and its metabolites (DDD, DDE), $\mathrm{\gamma HCH}$ and $\alpha \mathrm{HCH}$, aldrin, endrin, diéldrin, heptachlor, heptachlor epoxide, hexachlorobenzene (HCB) and a endosulfan.

The study showed an important contamination by organochlorine pesticides, DDT and its metabolites present the high concentrations at all species, lindane and its isomers are also present but at low concentrations. At clams, concentrations of POC varied between 9.2 to $51.72 \mathrm{ng} / \mathrm{g}$ (dry weight) with a maximum detected in the station of oued Drader. Mussels were less contaminated that clams, concentrations 
varied between 4.53 to $16.81 \mathrm{ng} / \mathrm{g}$. The contamination of mullet by POC is more marked with a concentrations varied between $12.52 \mathrm{ng} / \mathrm{g}$ recorded in November to $82.39 \mathrm{ng} / \mathrm{g}$ detected in May. The strong proportion of DDE in relation to DDT showed that the major of contamination is very old.

The quantities of organochlorine pesticides revealed at clams, mussels and mulled of Moulay Bousselham lagoon don't exceed the maximal limits in marines organisms. The consumption of these species don't have actually any problem in public health.

Keywords : Organochlorine, pesticides, bivalves, fish, lagoon, Morocco.

\section{Introduction}

Les pesticides organochlorés (POC) comme DDT (dichloro-diphenyltrichloroethanes) et $\mathrm{HCH}$ (hexachlorocyclohexanes), représentent un important groupe des Polluants Organiques Persistant (POP) qui sont connus pour être très toxiques, plus ou moins persistants dans l'environnement, bioaccumulables à travers la chaîne alimentaire et peuvent être transporté sur une longue distance [1-3].

Pendant des dizaines d'années, ces produits chimiques hautement toxiques ont fragilisé la santé des êtres humains et des animaux, endommageant leurs systèmes immunitaire, reproductif et nerveux [4]. Considérant, les problèmes écotoxicologiques et environnementaux liés à l'usages des pesticides organochlorés, les pays développés ont interdit leur usage dans tous les domaines depuis 1972 [5].

Au Maroc, ils ne sont interdits que depuis 1984, sauf pour le DDT et le lindane qui sont encore tolérés en santé publique [6]. Les stocks déjà disponibles après cette date constituaient alors des produits périmés. En outre, la législation marocaine reste insuffisante dans le domaine du contrôle des stocks, leurs conditions et la durée de stockage et le contrôle des formulations des matières actives. 
Les pesticides organochlorés sont largement utilisés durant la période 1960-1984 à la fois en agriculture et en santé publique pour éradiquer certaines maladies, ils ont été estimés à plus de 10000 tonnes utilisés par année [7].

Différents auteurs se sont intéressés à l'étude de la distribution des pesticides organochlorés dans l'environnement aquatique. Parmi les plus récents, l'on peut citer les travaux de [4,8-13]. La pollution par les pesticides organochlorés est relativement bien étudiée au niveau des côtes méditerranéennes marocaines (programme Medpol, [14,15]), alors que sur les côtes atlantiques ces études sont très rares. il y a une étude préliminaire qui a été réalisée au niveau de la lagune de Moulay Bousselham [16] et deux autres études effectuées au niveau des estuaires du Loukkos, Sbou, Bou-regreg et Oum Rbaa [17,18].

L'objectif de cette étude est d'évaluer le niveau de la contamination par les pesticides organochlorés des principales composantes de la chaîne trophique (mollusque bivalve et poisson) au niveau de la lagune de Moulay Bousselham (région du Gharb).

Cette évaluation est très importante vue la forte influence anthropique dans cette région à cause de l'activité urbaine, industrielle et surtout agricole très intense. En effet, le développement de l'activité agricole aux alentours de cette lagune, depuis quelques années, a entraîné l'introduction de nombreuses substances chimiques (fertilisants et pesticides, etc...).

\section{Matériel et méthodes}

\section{2-1. Site d'étude}

La lagune de Moulay Bousselham est située à $80 \mathrm{~km}$ au nord de Kénitra et $40 \mathrm{Km}$ au sud de Larache. C'est une cuvette elliptique (Figure 1) longue de $9 \mathrm{~km}(\mathrm{~N}-\mathrm{S})$, large de $5 \mathrm{~km}(\mathrm{E}-\mathrm{O})$ et d'une superficie de $30 \mathrm{Km}^{2}$ avec un volume variable suivant les marées et les saisons. 

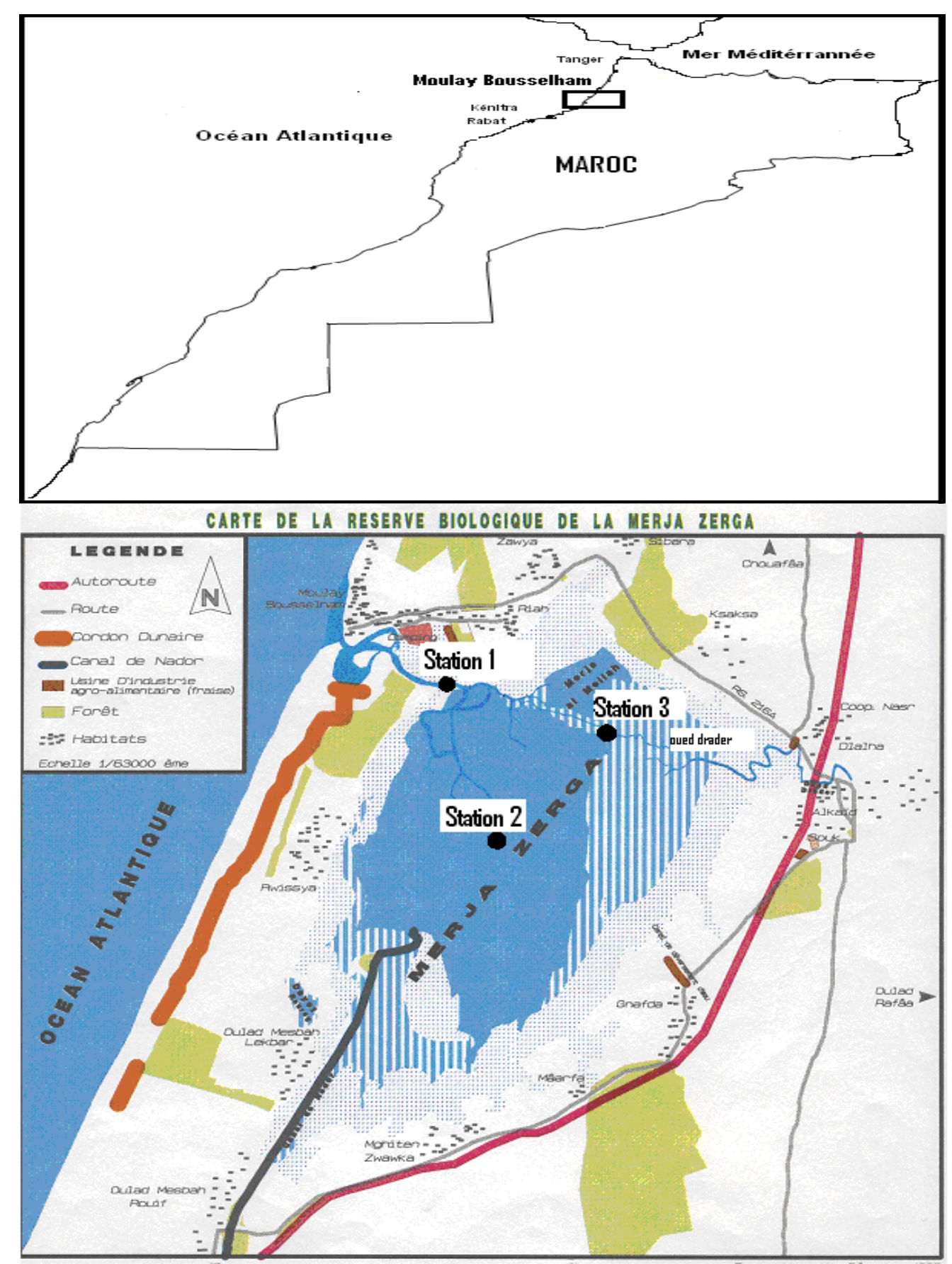

Figure 1 : Morphologie de la lagune de Moulay Bousselham et localisation des stations d'échantillonnage 
La communication avec la mer se fait par l'intermédiaire d'un goulet qui présente une alternance d'ouverture et de fermeture. La lagune est alimentée en eau douce par l'Oued Drader qui draine un bassin d'environ $1500 \mathrm{Km}^{2}$ au Nord-Est et le canal de Nador qui se déverse dans la partie Sud de la merja.

\section{2-2. Echantillonnage de la faune}

L'échantillonnage de la faune, la palourde (Venerupis deccusatua), moule (Mytilus edulis) et le poisson mulet (Mugil cephalus), s'est déroulée au cours de quatre campagnes de prélèvement février (hiver), mai (printemps), août (été) et novembre (automne) 2003.

Les échantillons de palourdes adultes, d'une taille la plus homogène possible ont été récoltés dans trois stations (Figure 1), sélectionnés en tenant compte de la répartition de l'espèce et de la position des sources de pollution par rapport au milieu récepteur.

Les échantillons de moules ont été prélevés uniquement au niveau de la station prés de goulet $S_{1}$. Les échantillons de mulet provenaient directement des prises de pêcheurs à l'intérieur de la lagune. C'est un poisson lagunaire qui s'alimente en broutant le long du fond, en avalant les dépôts et les détritus. Donc, il est exposé à la contamination du fait que son milieu est directement pollué par les déchets urbains et industriels.

\section{2-3. Prétraitement des échantillons}

Pour les échantillons de bivalves, les individus constituent un lot homogène en taille et reproductible d'un prélèvement à l'autre. Les coquillages sont triés puis stockées dans des sacs en plastiques contenant l'eau du milieu de prélèvement pour épuration. Après, les parties molles de 50 individus sont extraites des coquilles et elles sont mise à égoutter dans un entonnoir de Buchner, puis chaque échantillon est broyé et mis dans des piluliers pour être lyophilisé. 
Les poissons ont été regroupés par "pool de 10 " selon la taille. Le muscle en entier des poissons a été disséqué, homogénéisé et mise dans des piluliers pour être lyophilisé.

\section{2-4. Procédures d'analyse}

Les concentrations des pesticides organochlorés sont déterminées par la procédure décrite à UNEP/FAO/IOC/AIEA [19].

Les échantillons de biota sont décongelés et lyophilisés avant d'être analysés. Approximativement $10 \mathrm{~g}$ (Poids $\mathrm{sec}$ ) sont extraits avec n-hexane durant 8 heures au soxhlet. L'extrait est ensuite traité par l'acide sulfurique pour l'élimination des lipides. La séparation est réalisée par chromatographie sur colonne de Florisil. Ce dernier est activé dans le four à $130^{\circ} \mathrm{C}$ pendant 12 heures puis il est partiellement désactivé avec $0,5 \%$ d'eau distillée puis conservé dans des récipients en verre avant son utilisation.

Les résidus de pesticides organochlorés sont séparés et quantifiés par chromatographie en phase gazeuse VARIAN CP 3380 équipé d'un détecteur à capture d'électron $(63 \mathrm{Ni})$ et d'une colonne capillaire "non polaire » CP-Sil 8 CB de $30 \mathrm{~m} \times 0,25 \mathrm{~mm} \times 0,25 \mu \mathrm{m}$ (5\% diphenyl- $95 \%$ dimethylpolisiloxane).

L'injection a été réalisée en mode spliteless et le volume d'injection a été de $1 \mu \mathrm{L}$. La programmation de la température était comme suit : détecteur à $300^{\circ} \mathrm{C}$, injecteur à $280^{\circ} \mathrm{C}$ et Colonne: $70^{\circ} \mathrm{C}$ à $140^{\circ} \mathrm{C}$ à raison de $20^{\circ} \mathrm{C} / \mathrm{min}$, à $260^{\circ} \mathrm{C}$ à raison de $5^{\circ} \mathrm{C} / \mathrm{min}$.

L'identification d'un composé est fondée sur son temps de rétention qui correspond au temps de sortie du produit de la colonne. La teneur de l'échantillon en différents composés était quantifiée par la comparaison de la surface du produit correspondant dans la solution d'étalon. Une quantification externe a été réalisée par l'injection d'un mélange de composés étalons après toutes les deux injections d'extraits d'échantillons. 
Les solutions étalon mère ont été fournies par Promochem. La pureté des résidus varie entre 96 et $99,8 \%$.

Des blancs de procédures ont été analysés en même temps que nos échantillons de biota en les dopant avec une solution à $25 \mathrm{pg} / \mu \mathrm{L}$ de 2,4,5 trichlorobiphényl (PCB 29) et $20 \mathrm{pg} / \mathrm{\mu L}$ de $\varepsilon \mathrm{HCH}$ comme étalons internes. Le pourcentage de récupération de la méthode utilisée était compris entre 70 à $90 \%$ pour tous les composés. La validité des méthodes analytiques a été vérifiée en analysant des échantillons de référence de tissu des moules (IAEA- 432) [20].

La variabilité des teneurs des POC est étudiée par une analyse de variance à deux facteurs (saison et station) effectuée par la procédure ANOVA du logiciel SAS [21].

Douze pesticides organochlorés ont été recherchés: I'hexachlorobenzène, $\alpha$ endosulfan, DDT et ses métabolites (DDT, DDD, $\mathrm{DDE})$, Hexachlorocyclohexane et ses isomères $(\alpha \mathrm{HCH}$ et $\gamma \mathrm{HCH})$ et les cyclopentadiennes (Aldrine, dieldrine, endrine, heptachlore et heptachlore epoxide).

\section{Résultats et discussion}

Les concentrations moyennes des résidus des pesticides organochlorés chez les espèces étudiées de la lagune de Moulay Bousselham sont présentées dans le Tableau 1.

Les analyses ont montré que la plupart des espèces étudiées sont contaminées et que les niveaux de contamination varient selon les composés. Parmi les composés recherchés, le DDT et ses métabolites $\left(\mathrm{DDT}_{\mathrm{T}}\right)$ sont les plus couramment détectés et présentent des teneurs plus élevées par rapport aux autres composés.

Les composés cyclopentadiènes (aldrine, endrine, dieldrine, heptachlor et heptachor epoxide), interdits depuis 1984 au Maroc, ne sont présents qu'à l'état de trace ou sont même absents dans certaines espèces, ce qui implique que l'exposition à ces produits a cessé. 
Tableau 1: Teneurs moyennes des pesticides organochlorés ( $\mathrm{ng} / \mathrm{g}$ poids sec) détectées chez les espèces récoltées au niveau de la lagune de Moulay Bousselham.

\begin{tabular}{|c|c|c|c|}
\hline $\begin{array}{l}\text { Espèces } \\
\text { Composés }\end{array}$ & $\begin{array}{c}\text { Palourde } \\
\text { (Venerupis deccusatua) }\end{array}$ & $\begin{array}{c}\text { Moule } \\
\text { (Mytilus edulis) }\end{array}$ & $\begin{array}{c}\text { Mulet } \\
\text { (Mugil cephalus) }\end{array}$ \\
\hline 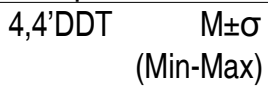 & $\begin{array}{c}4.90 \pm 1.91 \\
(2.15-10.25)\end{array}$ & $\begin{array}{l}2.18 \pm 1.45 \\
(1.25-4.35)\end{array}$ & $\begin{array}{c}4.69 \pm 2.67 \\
(2.20-8.48)\end{array}$ \\
\hline 4,4'DDE & $\begin{array}{c}7.75 \pm 1.25 \\
(2.50-20.15)\end{array}$ & $\begin{array}{l}2.63 \pm 2.10 \\
(1.60-6.18)\end{array}$ & $\begin{array}{l}21.47 \pm 19.32 \\
(2.78-48.65)\end{array}$ \\
\hline 4,4'DDD & $\begin{array}{c}5.67 \pm 1.65 \\
(2.14-13.27)\end{array}$ & $\begin{array}{l}2.81 \pm 1.24 \\
(2.13-4.68)\end{array}$ & $\begin{array}{c}8.58 \pm 6.29 \\
(2.14-15.51)\end{array}$ \\
\hline$\gamma \mathrm{HCH}$ & $\begin{array}{l}2.86 \pm 1.32 \\
(1.12-6.20)\end{array}$ & $\begin{array}{l}1.10 \pm 1.35 \\
(\mathrm{nd}-2.77)\end{array}$ & $\begin{array}{l}2.40 \pm 2.1 \\
(\mathrm{nd}-5.1)\end{array}$ \\
\hline$\alpha \mathrm{HCH}$ & $\begin{array}{c}0.21 \pm 0.24 \\
(n d-1.9)\end{array}$ & nd & $\begin{array}{l}0.84 \pm 1.04 \\
\text { (nd }-2.28)\end{array}$ \\
\hline Aldrine & $\begin{array}{l}1.13 \pm 0.43 \\
(n d-3.45)\end{array}$ & nd & $\begin{array}{l}0.64 \pm 0.42 \\
\text { (nd }-0.98)\end{array}$ \\
\hline Endrine & $\begin{array}{l}0.6 \pm 0.17 \\
(n d-0.75)\end{array}$ & nd & $\begin{array}{c}0.30 \pm 0.35 \\
(\mathrm{nd}-0.71)\end{array}$ \\
\hline Dieldrine & nd & nd & $\begin{array}{l}0.66 \pm 0.53 \\
(n d-1.25)\end{array}$ \\
\hline Heptachlor & $\begin{array}{l}0.50 \pm 1.25 \\
(n d-1.75)\end{array}$ & nd & $\begin{array}{c}0.89 \pm 0.63 \\
(0.22-1.75)\end{array}$ \\
\hline Heptachlor époxide & $\begin{array}{l}0.2 \pm 0.37 \\
(n d-0.4)\end{array}$ & nd & $\begin{array}{l}0.31 \pm 0.37 \\
(n d-0.85)\end{array}$ \\
\hline $\mathrm{HCB}$ & $\begin{array}{l}1.81 \pm 0.56 \\
(n d-5.75)\end{array}$ & nd & $\begin{array}{l}1.05 \pm 0.70 \\
(\mathrm{nd}-1.54)\end{array}$ \\
\hline$\alpha$ Endosulfan & nd & nd & nd \\
\hline POC & 25.63 & 8.72 & 41.83 \\
\hline
\end{tabular}

POC : Pesticides organochlorés totaux

$$
\begin{aligned}
& M \text { : moyenne } \\
& \sigma \text { : écart-type }
\end{aligned}
$$

Min-Max : Minimum - maximum 


\section{3-1. Contamination des palourdes}

Les analyses ont montré la présence des molécules de pesticides organochlorés dans les palourdes au niveau de trois stations de la lagune à des doses décelables, et que les niveaux de contamination varient selon les composés (Tableau 1).

Parmi les composés recherchés, le DDT et ses métabolites (DDT $)$ ainsi que le lindane $(\gamma \mathrm{HCH})$ sont les plus couramment détectés et présentent des teneurs plus élevées par rapport aux autres composés au niveau des trois stations. Les valeurs du DDT varient entre 7.55 et $43.68 \mathrm{ng} / \mathrm{g} \mathrm{du}$ poids sec et celles du lindane entre 1.20 et $6.20 \mathrm{ng} / \mathrm{g}$.

Les autres composés ne sont pas décelés dans la plupart des échantillons et sont en général à l'état de traces (Tableau 1). Cette faible contamination par ses composés peut être due au faite que ces composés ont été interdits au Maroc depuis 1984. Mais le fait qu'ils sont encore détectés pourrait être expliqué par l'utilisation illégale de certains stocks anciens de ces composés dans la région du Gharb [22].

Le DDT est peu soluble dans l'eau et il est très fortement bioaccumulable dans les tissus lipidiques des organismes aquatiques [23].

Le $\gamma \mathrm{HCH}$ (lindane) était omniprésent dans tous les échantillons mais à des faibles quantités.

En effet, le lindane est beaucoup plus soluble dans l'eau et il présente un caractère moins lipophile que le DDT et par conséquent il s'accumule moins dans les organismes aquatiques [24].

L'analyse spatiale des teneurs en pesticides organochlorés au niveau des palourdes de la lagune de Moulay Bousselham montre que c'est la station $S_{1}$ qui abrite les concentrations les plus faibles, alors que les teneurs les plus élevées sont enregistrées au niveau des palourdes des stations $S_{2}$ et $S_{3}$ (Figure 2). Les faibles teneurs enregistrées au niveau de $S_{1}$ peuvent être expliquées par le fait que cette station, située à l'aval de la lagune, se trouve à l'abri de tous rejets et elle est soumise aux jeux des marées, ce qui semble perturber le piégeage des pesticides par les 
sédiments au sein de ce milieu très agité. Les concentrations les plus élevées enregistrées au niveau des palourdes des stations $S_{2}$ et $S_{3}$ sont dues surtout aux apports des polluants de l'oued Drader et du canal du Nador. Ces différences des niveaux de contamination selon les stations semblent liées aux différences en teneurs en lipides [25].

Ce sont les palourdes des stations $S_{2}$ et $S_{3}$ qui contiennent plus de lipides (3.5 à $5.6 \%$ de lipides) donc qui accumulent plus des composés organochlorés.

Le niveau de contamination des palourdes par les POC et en particulier du DDTs a présenté une variation temporelle (Figure 2), les concentrations les plus élevées sont obtenues au mois de mai (printemps) et les plus faibles au mois novembre (automne) et ceci pour les trois stations. Alors que les teneurs en lindane ne présentent aucune variation spatio-temporelle.

En effet, La croissance et le développement des bivalves dépendent de l'intensité du stockage des matières de réserves qui fluctuent saisonnièrement pour des raisons physiologiques. Elle est maximale avant la reproduction et minimal après la ponte lorsque les réserves ont été épuisées au cours de la gamétogenèse [26]. Au Maroc, sous un climat tempéré, la reproduction des palourdes a lieu au printemps [27].

L'analyse de variance effectuées sur les teneurs des pesticides organochlorés $(\mathrm{POC})$ et du DDTs chez les palourdes révèle à travers le test $F$ des différences significatives $(P<0.05)$ entre les stations et les saisons (Tableau 2).

Tableau 2 : Analyse de variance à deux variables pour les pesticides organochlorés totaux (POC), DDTs et le lindane au niveau des palourdes (test $F$ significatif si $P<0.05$ ).

\begin{tabular}{cccc}
\hline & POC & DDTs & Lindane \\
\hline Saisons & 0.0035 & 0.0056 & 0.2498 \\
Stations & 0.0084 & 0.0069 & 0.3042 \\
\hline
\end{tabular}


Par ailleurs, la comparaison des variances sur les teneurs du lindane ne révèle en revanche aucune différence significative entre les palourdes des différentes stations, ni entre les saisons.

Le niveau de contamination des palourdes par le DDT est très bas en comparaison avec les données rapportées par Mehdaoui et al. [16] qui ont observé des concentrations de DDTs qui varient de 10 à $107 \mathrm{ng} / \mathrm{g}$. Alors que les concentrations des autres composés sont comparables à celles obtenues par la présente étude. Les valeurs maximales étaient de $8.64 \mathrm{ng} / \mathrm{g}$ pour le lindane, $7.21 \mathrm{ng} / \mathrm{g}$ pour le $\alpha \mathrm{HCH}$ et $4 \mathrm{ng} / \mathrm{g}$ pour le HCB.
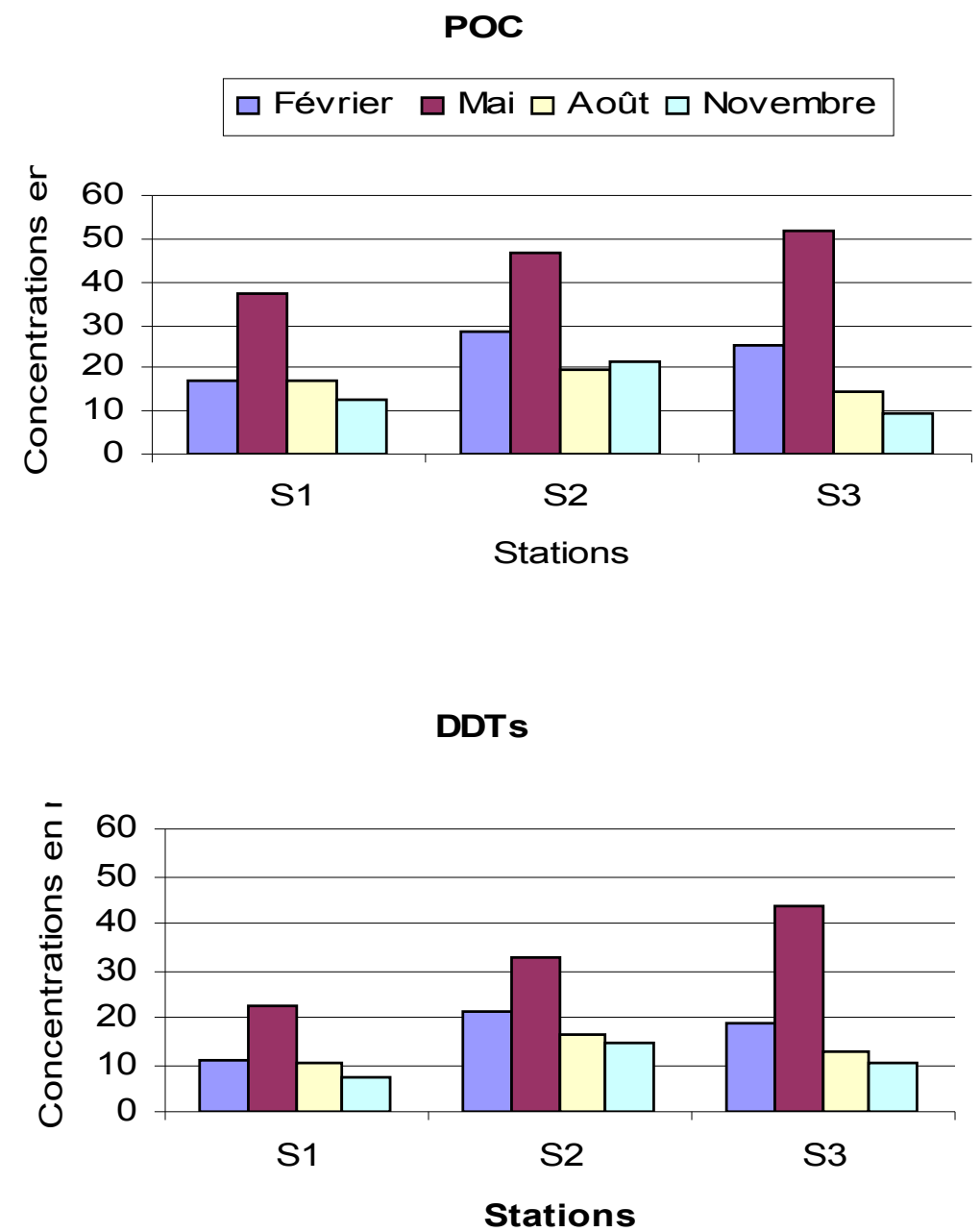

Benbakhta Bouchaib et al. 


\section{Lindane}

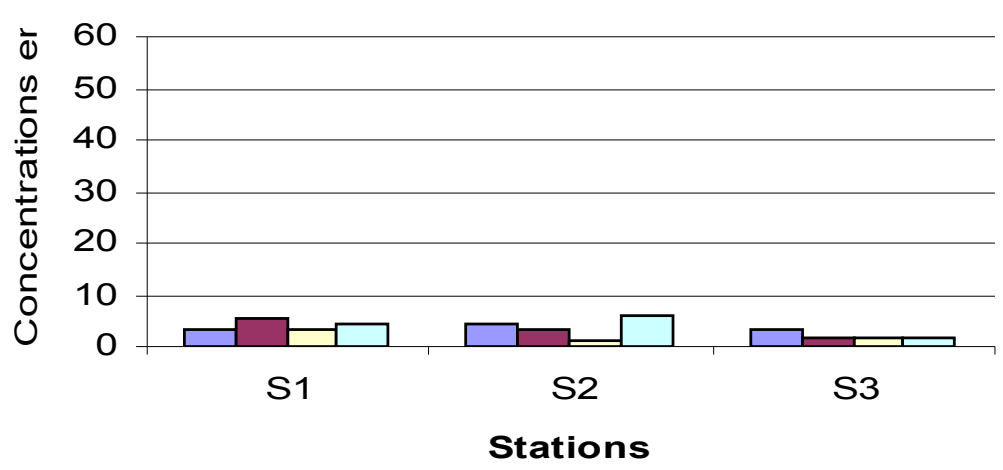

Figure 2 : Variations saisonnières des teneurs des pesticides organochlorés chez les palourdes de la lagune de Moulay Bousselham.

La composition moyenne des isomères de DDT chez les palourdes est de $18 \%$ pour DDT, $49 \%$ pour DDE et de $13 \%$ pour DDD (Figure 3 ). On remarque que le DDE est plus abondant que le DDD et le DDT. Cette forte proportion de DDE, principal métabolite de DDT, indique que l'utilisation de DDT est très ancienne.

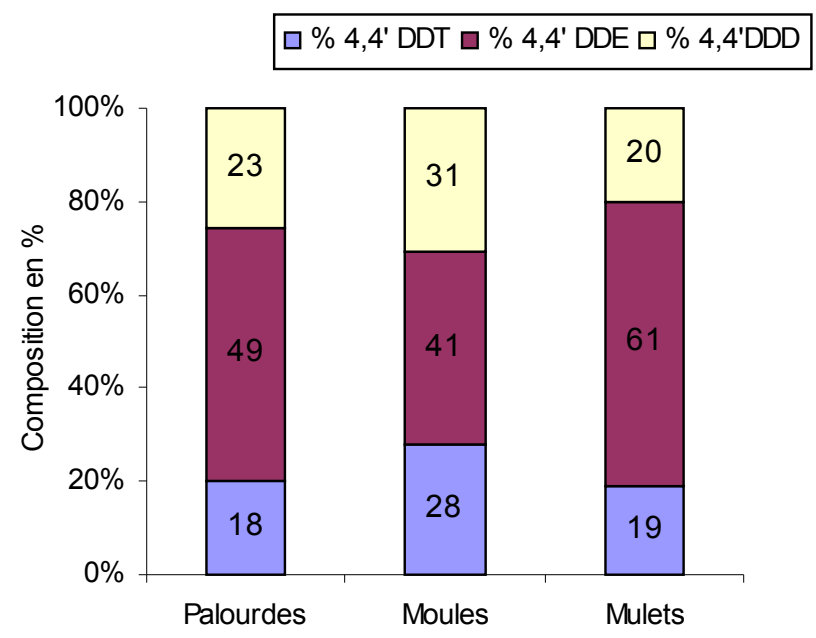

Figure 3 : Composition des isomères de DDT chez les espèces étudiées. 


\section{3-2. Contamination des moules}

Les concentrations moyennes en insecticides organochlorés détectés dans les tissus de moules figurent dans le Tableau 1.

On a décelé que les isomères de DDT et le lindane. Alors que les composés de groupe des cyclopentadiènes n'ont pas été décelés dans tous les échantillons. Ceci confirme la cessation de l'utilisation de ces composés organochlorés qui ont été interdits dans notre pays depuis 1984.

Les moules sont faiblement contaminés, les teneurs varient de 1.25 à 4.35 pour p,p'DDT; de 1.60 à 6.18 pour p,p' DDE et de 2.13 à $4.68 \mathrm{ng} / \mathrm{g}$ pour $p, p^{\prime} D D D$.

Le niveau de contamination des moules par les POC a présenté une variation temporelle (Figure 4), les concentrations les plus élevées, en particulier de DDT total, sont obtenues au mois de mai et les plus faibles au mois d'août et novembre.

$\square$ POC $\square$ DDTs $\square \mathrm{HCHs}$

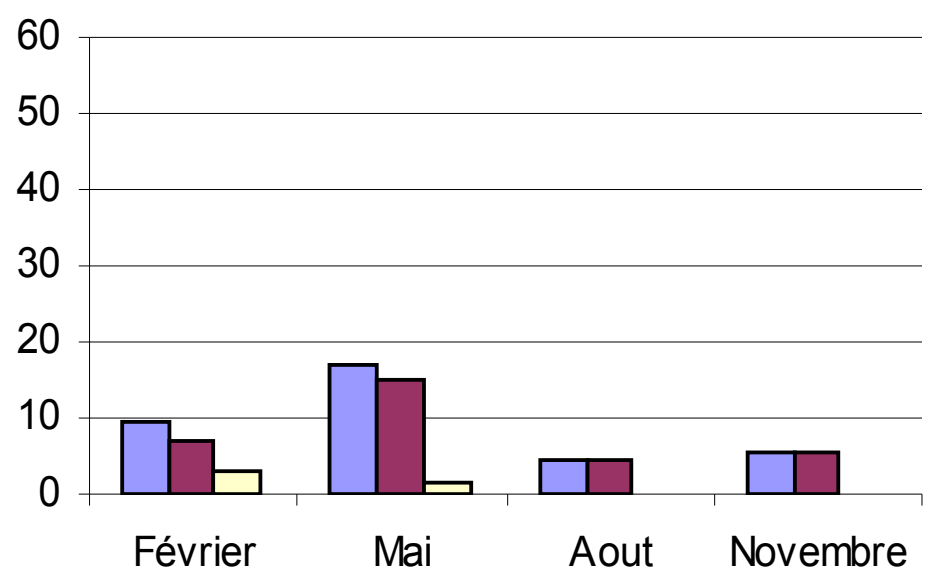

Figure 4 : Variations saisonnières des teneurs des pesticides organochlorés chez les moules de la lagune de Moulay Bousselham 
Cette différence est due surtout au contenu lipidique des moules qui peut subir des variations importantes selon l'état physiologique et les conditions du milieu [25].

De même le DDE était nettement plus abondant que le DDD et le DDT, il représentait un peu près $41 \%$ du DDT total (Figure 3).

La comparaison des teneurs de DDT total avec celle relevées dans d'autres régions du monde (Tableau 3) montre que le niveau de contamination des bivalves de la lagune de moulay Bousselham s'est avérée très bas $(7.62-18.32 \mathrm{ng} / \mathrm{g})$. Nos résultats sont comparables avec ceux détectés dans les bivalves du littoral méditerranéen marocain, des côtes norvégiens et du golf du Oman. Alors qu'en Egypte et en en Asie (Hong Kong, Philippine) où les pesticides organochlorés, et spécialement le DDT, ont été extrêmement utilisés en agriculture et en santé publique, les concentrations du DDT total dans les bivalves étaient plus élevées que celles de la présente étude.

Tableau 3 : Comparaison des teneurs moyennes de DDT total ( $\mathrm{ng} / \mathrm{g}$ ) dans nos échantillons de bivalves avec celles relevées dans d'autres études

\begin{tabular}{|l|l|l|l|}
\hline \multicolumn{1}{|c|}{ Pays } & \multicolumn{1}{|c|}{ Espèce } & \multicolumn{1}{c|}{ R DDT } & \multicolumn{1}{c|}{ Référence } \\
\hline Littoral méditerranéen, Maroc & Mytilus edulis & 19.34 & Alami,1991 [14] \\
Mersey estuary, England & Mytilus edulis & $19-74$ & Connor et al., 2001 [28] \\
Tokyo Bay, Japan, Japan & Mytilus galloprovincialis & $3.5-52$ & Monirith et al., 2003 [29] \\
Coastal waters of Xiamen China & Mytilus edulis & 133 & Klumpp et al., 2004 [30] \\
Norvegian coast & Mytilus edulis & $21-30$ & Green et Knutzen, 2003 [31] \\
Gulf of Naples, Italy & Mytilus galloprovincialis & 177.2 & Naso et al., 2005 [32] \\
Adriatic Sea, Italy & Mytilus galloprovincialis & 141.7 & Stefanelli et al., 2004 [33] \\
Tomales Bay, California & Mytilus edulis & 15 & NOAA,1989 [34] \\
Missions Bay, California & Mytilus edulis & 70 & NOAA,1989 [34] \\
Pearl River Estuary, China & Perna virdis & 51.4 & Fu et al., 2003 [35] \\
Philippines & Perna virdis & $14-200$ & Monirith et al., 2003 [29] \\
Hong Kong & Perna virdis & $33-4348$ & Monirith et al., 2003 [29] \\
Egyptian Red Sea coast & Brachiodantes sp & $543-3356$ & Khaled et al., 2004 [36] \\
Gulf of Oman & Oystrea sp & $09-4.8$ & Mora et al., 2005 [37] \\
Lagoons of Ghana & Perna parna & 73 & Otchere., 2005 [38] \\
Lagune My Bousselham, Maroc & Mytilus edulis & 7.62 & Présente étude \\
Lagune My Bousselham, Maroc & Venerupis deccusatua & 18.32 & Présente étude \\
& \multicolumn{2}{|l}{} \\
\hline
\end{tabular}




\section{3-3. Contamination des mulets}

Les échantillons de mulet (Mugil cephalus) sont beaucoup plus contaminés que les palourdes principalement par le DDT et ses métabolites, les teneurs varient de 2.20 à 8.48 pour p,p'DDT; de 2.78 à 48.65 pour p,p' DDE et de 2.14 à $15.51 \mathrm{ng} / \mathrm{g}$ pour p,p'DDD. Cette abondance des résidus de DDT peut résulter des processus de métabolisme ou biodégradation subit par ces substances, en effet le DDT à une durée de rémanence plus élevée par rapport aux autres composés, ce qui le rend difficilement biodégradable. Par ailleurs, sa prédominance pourrait être expliquée par une éventuelle utilisation intensive dans le domaine de la santé.

La variation temporelle des teneurs en pesticides organochlorés chez le mulet de la lagune de Moulay Bousselham montre une différence bien marquée entre les quatre périodes (Figure 5), ainsi les valeurs les plus élevées ont été enregistrés durant le mois de mai avec $82.39 \mathrm{ng} / \mathrm{g}$. Les valeurs les plus faibles ont été trouvés au mois de novembre avec 12.52 $\mathrm{ng} / \mathrm{g}$.

L'analyse de variance effectuées sur les teneurs des pesticides organochlorés (POC) et du DDTs chez les mulets révèle à travers le test $F$ des différences significatives $(P<0.05)$ entre les saisons (Tableau 4). Par ailleurs, la comparaison des variances sur les teneurs du lindane ne révèle en revanche aucune différence significative entre les mulets des différentes saisons.

Tableau 4 : Analyse de variance à deux variables pour les pesticides organochlorés totaux (POC), DDTs et le lindane au niveau des Mugil cephalus (test $F$ significatif si $P<0.05$ )

\begin{tabular}{llll}
\hline & POC & DDTs & Lindane \\
\hline Saisons & 0.0028 & 0.0064 & 0.2647 \\
\hline
\end{tabular}


L'évolution du cycle lipidique pourrait être responsable des variations des teneurs des composés organochlorés. Dans tous les cas, une augmentation du contenu du poisson en lipides est accompagnée d'une élévation en composés organochlorés en particulier de DDT qui a un caractère plus lipophile.

De même que chez les palourdes, Le DDE est nettement plus abondant que le DDD et le DDT; il représentait plus de $60 \%$ de DDT total (Figure 3). Cette forte proportion de DDE par rapport au DDT total chez le mulet suggère que l'accumulation résulte d'une exposition directe de DDE dans l'environnement ou dans l'alimentation. Mais le fait que le DDT soit encore observé chez les organismes aquatiques ne peut être dû qu'à un apport récent de ce composé dans le système lagunaire [12]. Au contraire, les composés cyclodiènes sont faiblement ou pas du tout accumulé chez le mulet, ce qui confirme que l'exposition à ces composés a cessé.

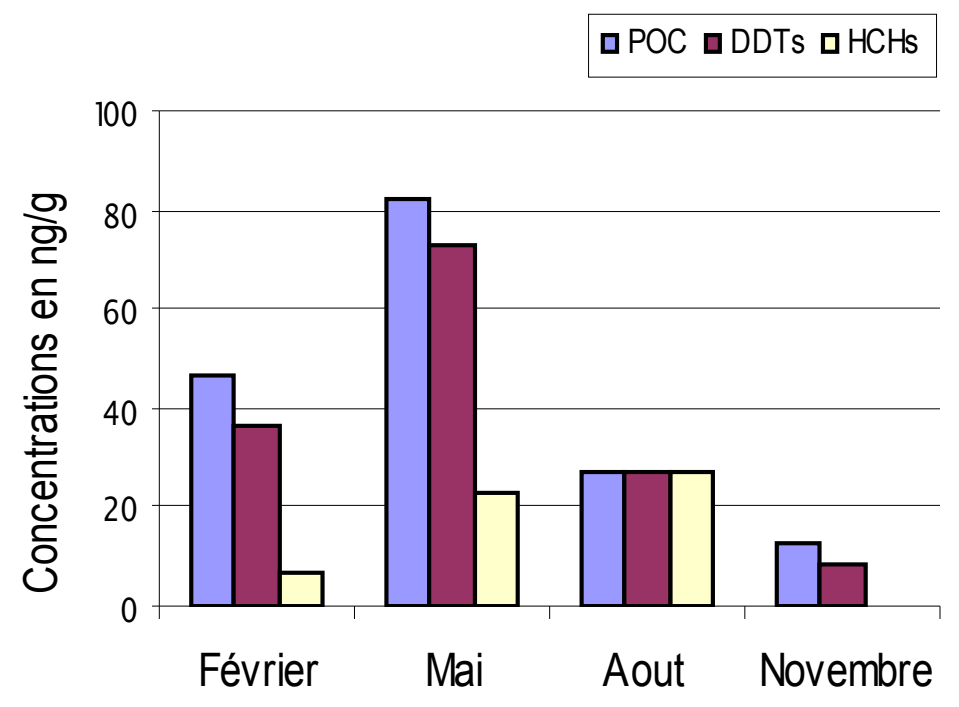

Figure 5 : Variations saisonnières des teneurs des pesticides organochlorés chez l'espèce Mugil cephalus de la lagune de Moulay Bousselham. 
Le niveau de contamination de mulet de la lagune de Moulay Bousselham est trop bas en comparaison avec les données d'autres études. Des teneurs moyennes du DDT total détectées chez Mugil cephalus étaient de $703.3 \mathrm{ng} / \mathrm{g}$ au golf du Naples, Italie [32], de $388 \mathrm{ng} / \mathrm{g}$ à Embro delta, Espagne [39].

\section{3-4. Evaluation du risque sur la santé}

Les niveaux des pesticides organochlorés détectés chez les différentes espèces étudiées sont très inférieurs aux limites maximales des résidus (LMR) pour les produits de la pêche (Tableau 5).

L'évaluation du risque pour la santé humaine est faite en utilisant la méthode de comparaison de l'apport quotidien en pesticides organochlorés chez les gros consommateurs de poissons (150g/j) avec les valeurs quotidiennes admissibles recommandées par Codex Alimentarius pour un poids corporel de $70 \mathrm{~kg}$.

L'apport quotidien admissible (AQA) exprime la dose, exprimé en fonction du poids corporel, d'une substance qui peut être toléré sur une durée de vie sans risque appréciable pour la santé.

Les apports quotidiens admissibles recommandés par Codex Alimentarius pour un poids corporel de $70 \mathrm{~kg}$ sont présentés dans le Tableau 5.

On remarque que les valeurs des $A Q A$ de la présente étude sont très inférieures à ceux fixés par le comité d'experts du Codex Alimentarius (Tableau 5). On note également comment la consommation de ces espèces contenant ces composés des pesticides organochlorés contribue à l'apport quotidien admissible de gros consommateur de poissons. Le taux de contribution varie de 0.07 à $3.6 \%$. La contribution de l'heptachlor et de l'aldrine à son apport quotidien est la plus grande, alors que celle de DDTs, lindane, endrine et HCB n'atteints pas le taux de $1 \%$.

Ainsi le risque de la consommation des poissons de la lagune reste limité uniquement dans le cas où il n'existe pas d'autre sources d'exposition. 
Tableau 5 : Comparaison de l'apport quotidien admissible (AQA) avec l'apport quotidien de gros consommateurs (AQGC) de poissons (150g/j)

\begin{tabular}{|c|c|c|c|c|c|}
\hline Pesticides & $\begin{array}{c}\mathrm{LMR}^{1} \\
\mu \mathrm{g} / \mathrm{kg} \\
\text { poids frais }\end{array}$ & $\begin{array}{c}\text { AQA }^{2} \\
\mu \mathrm{g} / \text { personne } \\
\text { /jour }\end{array}$ & $\begin{array}{l}\text { Concentrations } \\
\text { poissons } \\
(\mu g / \mathrm{Kg})\end{array}$ & \multicolumn{2}{|c|}{$\begin{array}{c}\text { AQGC } \\
\mu \mathrm{g} / \text { personne/jour } \\
\% \mathrm{AQA}\end{array}$} \\
\hline DDTs & 200 & 1200 & 34.75 & 5.2 & 0.43 \\
\hline Lindane & 100 & 480 & 2.40 & 0.36 & 0.07 \\
\hline Aldrine & 200 & 7.0 & 1.30 & 0.19 & 2.71 \\
\hline Endrine & 10 & 6.0 & 0.30 & 0.04 & 0.66 \\
\hline HCB & 200 & 42 & 1.05 & 0.15 & 0.35 \\
\hline Heptachlor & 200 & 5.0 & 1.20 & 0.18 & 3.60 \\
\hline
\end{tabular}

\section{Conclusion}

Les résultats obtenus montrent que les niveaux de contamination des organismes aquatiques de la lagune de Moulay Bousselham par les pesticides organochlorés restent globalement faibles et très inférieurs à ceux des autres études. Les teneurs sont plus élevées chez les poissons que chez les bivalves. Cependant, elles sont au dessous des niveaux maximales des résidus permis dans la communauté européenne. Le risque encouru par le consommateur de ces produits de la mer reste très faible. Néanmoins, un programme de surveillance régulière doit être établi afin de limiter au mieux la contamination de l'environnement marin par ces substances toxiques. 


\section{Références}

[1] - L. Guzzella, C. Rosscioli, L. Vigano, M. Saha, S.K. Sarkar and A. Bhattacharya. "Evaluation of the concentration of $\mathrm{HCH}, \mathrm{DDT}, \mathrm{HCB}$, PCB and PAH in the sediments along the lower stretch of Hugli estuary", West Bengal, northeast India. Environmental International 31(4) (2005) 523-534.

[2] - O. Wurl and J. P. Obbard. "Organochlorine pesticides, polychlorinated biphenyls and polybrominated diphenyl ethers in Singapore's coastal marine sediments". Chemosphere 58(7) (2005) 925-933.

[3] - S. Tanabe, R. Tatbukawa and H. Hidaka "Global distribution and atmospheric transport of chlorinated hydrocarbons: $\mathrm{HCH}(\mathrm{BCH})$ isomers and DDT compounds in the Western Pacific, eastern India and Antarctic oceans". J. Oceanogr. Soc. Jpn. 38 (1982) 137-148.

[4] - D. D. Nhan, F. P. Carvalho, N. Am Manh, N. Qooc Tuan, N. Thi hai yen, J. P. Villeneuve and C. cattini "Chlorinated pesticides and PCBs in sediments and molluscs from freshwater canals in the Hanoi region". International Pollution 112 (2001) 311-320.

[5] - Y. Sapozhnikova, O. Bawardi and D. Schlenk. "Pesticides and PCBs in sediments and fish from the Saton Sea, California, USA". Chemosphere 55 (2004) 797-809

[6] - MARA - Arrêté du Ministre d'Agriculture et de Réforme Agraire relatif à l'interdiction des pesticides organochlorés, № 466 (1984), Rabat.

[7] - ODI "L'industrie des pesticides au Maroc : situation actuelle et perspective d'avenir ". Colloque de l'ANAPAV sur les pesticides agricole, (1984) Rabat

[8] - M. I. Badawy and M. A. ElDib. "Residue of organochlorine pesticides in fish from Egyptian Delta lakes". Environ. Int. J, 10 (1984) 3-8.

[9] - S. M. Allen Gil, C. P. Gubala, R. Wilson, D. H. Landres, T. L. Wade, J. L. Sericano and L. R. Curtis. "Organochlorine pesticides and polychlorinated biphenyls (PCBs) in sediments and biota from US Arctic lakes". Arch Environ Contam. Toxicol. 33 (1997) 378-387.

[10] - KE Bernt, MO Hammill, M Lebeuf and KM Kovacs. "Levels and patterns of PCB and OC pesticides in harbour and grey seals the St Lawrence Estuary", Canada. Sci. Total Environ. 243-244 (1999) 243-262 
[11] - C. A. Bishop and J. D. Rouse. "Chlorinated hydrocarbon concentrations in plasma of the lake Erie water snake (Nerodia sipedon insularum) and northern water snake (Nerodia sipedon sipedon) from the Great Lakes basin in 1998". Arch. environ. Contam. Toxicol. 39 (2000) 500-505.

[12] - M. S. Abassy, H. Z. Ibrahim, H. M. Abdelkader. «Persistent Organochlorine Pollutants in the Aquatic Ecosystem of Lake Manzala", Egypt. Bull Environ Contam. Toxicol., 70 (2003) 1158-1164

[13] - S. Bayen, G. O. Thomas, H. K. Lee and J. P. Obbard "Organochlorine pesticides and heavy letals in green mussel, Perna virilis in Singapore». Water, Air, Soil Pollut. 155 (2004)103-116

[14] - M. Alami - Toxicité des pesticides organochlorés chez les organismes aquatiques: aspects environnementaux et expérimentaux. Thèse de 3e cycle, Fac. Sci. Rabat, (1991) 173p.

[15] - A. El Hraiki - Assessment of chlorinated hydrocarbons of trace metals contamination of Moroccan marine species. Thèse Doc. Vét., Institut Agronomique et Vétérinaire Hassan II, (1992) 135p.

[16] - O. Mehdaoui, M. Fekhaoui et C. descoins - Accumulation et biomagnification des insecticides organochlorés dans les mollusques et les poissons de la lagune de Moulay Bousselham, Au Maroc, Cahier santé, 10 (2000) 373-379

[17] - M. Boussaid - Résidus des pesticides organochlorés chez les organismes marins de la côte Atlantique Marocaine. Thèse Doc. Vét., Institut Agronomique et Vétérinaire Hassan II, (1994) 125p.

[18] - H. Fathi - Résidus de pesticides organochlorés chez des organismes aquatiques en provenance de quatre oueds marocains : Sebou, Bouregreg, Loukkos et Oum -Erabia, Thèse de Doctorat vétérinaire, Institut agronomique et Vétérinaire Hassan II, (1995) 111p.

[19] - UNEP/FAO/IOC/AIEA- Sampling of selected marine organisms and sample preparation for the analysis of chlorinated hydrocarbons, 12 (1991) 61-83.

[20] - J. P. Villeneuve, S. J. de Mora and C. Cattini - World-wide and regional intercomparison for determination of organochlorine compounds and petroleum hydrocarbons in mussels tissue IAEA-432, 74 (2004) $123 p$ 
[21] - D. Beauvillier - Programmes d'application SAS pour l'analyse des données. Quebec. SMG, (1995) 57-130.

[22] - PNUD - Gestion des polluants organiques persistents au Maroc, Projet POP (2004) 132p

[23] - A. Abarnou et V. Loizeau - La bioaccumulation : l'exemple des PCB. Océanis 20 (1994) 29-45.

[24] - J. C. Marchand, M.A. Caprais et P. Cosson Mannevy - Apports et distribution des résidus organochlorés à haut poids moléculaire dans la rade de Brest (milieu marin semi fermé). Oceanol. Acta 6 (1983) 269-292.

[25] - M. S. Galindo-Bect and P. flores-Base - DDT in Mytilus edulis : spatiotemporel variations in Punta Banda estuary, Baja California, Mexico. Bull. Env. Contam. Toxic., 46 (1991) 179-184.

[26] - UNEP/FAO/OMS/AIEA- Assessment of the state of pollution of the Mediterranean Sea by organohalogen compounds. MAP Technical Reports Series, 39 (1990) 105-216.

[27] - N. Rharbi - Essai d'élevage de la palourde Ruditapes deccussatus (L) dans la lagune de Moulay Bousselham (Côte atlantique marocaine). Thèse $3^{\text {ème }}$ cycle, Fac. Sci. Rabat, (1990) 146p.

[28] - L. Connor, M. S. Johnson, D. Copplestone and R. T. Leat-Recent trends in organochlorine residues in mussels (Mytilus edilus) from the Mersey estuary. Marine Environmental Research, 52 (2001) 397-411

[29] - Monirith I, Ueno D, Takahashi S, Nakata H, Sudaryanto A, Subramanian A, Karuppiah S., A. Ismail, M Mushtar and J. ZhengAsia Pacific mussel watch : monitoring contamination of persistent organochlorine compounds in coastal waters of Asian countries. Mar. Pollut. Bull., 46 (2003) 281300.

[30] - D. W. Klumpp, H. Hong, C. Humphrey, X. Wang and S. Codi - Toxic contaminants and their biological effects in coastal waters of Xiamen, China. Organic pollutants in mussel and fish tissues. Mar. Pollut. Bull., 44 (2002) $752-760$

[31] - N. W. Green and J. Kuntzen-Organohalogens and metals in marine fish and mussels ands some realtionships to biological variables at reference localities in Norway. Mar. Pollut. Bull., 46 (2003) 362-377.

[32] - B. Naso, D. Perrone, M.C. Ferrante, M. Bilancione and A. Lucisano Persistent organic pollutants in edible marine species from the Gulf of Naples, southern Italy. Science of total environment, 343 (2005) 83-95. 
[33] - P. Stefanelli, A. Di Muccio, F. Ferrara, D. Attard Barbini, T. Generali, P. Pelosi, G. Amendola, F. Vanni, S. Di Muccio and A. Ausili,Estimation of intake of organochlorine pesticides and chlorobiphenyls through edible fishes from the Italian Adriatic Sea during 1997. Food Control, 15 (2004) 27-38

[34] - NOAA - National status and trends program for marine environmental quality progress report: A summary of data on tissue contamination from the first three years (1986-1988) of the mussel wath project. national oceanic and atmospheric and administration. Tech. Memo. NOS OMA 49, NOAA/NOS/ Coastal and Estuarine Assessment Branch, Rockville, MD. (1989) $122 \mathrm{p}$.

[35] - J. Fu, B. mai, G. Sheng, X. Wang, P. Peng et al. - Persistent organic pollutants in environment of Oearl. river delta, China : an overview. Chemosphere, 52 (2003) 1411-1422.

[36] - A Khaled., A. El Nemr, T. O. Said, A. El-Sikaily and A. M. A. Abd-AllaPolychlorinated biphenyls and chlorinated pesticides in mussels from the Egyptian Red Sea coast. Chemosphere, 54 (2004) 1407 - 1412

[37] - S. de Mora, S. W. Fower, I. Tolosa, J. P. Vileneuve and C. Cattini, Chlorinated hydrocarbons in marine biota and coastal sediments from the Gulf and Gulf of Oman. Mar. Pollut. Bull., 50 (2004) 835-849

[38] - F. A. Otechere - Organochlorines (PCBs and pesticides) in the bivalves Anadara (Senilis) senilis, Crassostrea tulipa and Perna perna from the lagoons of Ghana. Science of Total Environment, 348 (2005) 102114.

[39] - D. Pastor, J. Boix, v. Fernandez and J. Albaigés- Bioaccumulation of organochlorinated contaminants in three estuarine fish species (Mullus barbatus, Mugil cephalus and Dicebtrarchus labratus). Mar. Pollut. Bull.; 32 (1996) $257-262$.

[40] - Directive Européenne. Règlement $C E n^{\circ} 22$ portant fixation de teneurs maximales pour certains contaminants dans les denrées alimentaires, (2001) 20p.

[41] - Codex Alimentarius - Joint FAO/WHO Food Standard Program. Vol. 2B. Residues in Food. FAO/WHO, Rome, (2005) 61-81. 